\title{
A criterion for local embeddability of three-dimensional CR structures
}

\author{
Gerd Schmalz $^{1}$ (D) Masoud Ganji ${ }^{1}$
}

Received: 12 April 2018 / Accepted: 1 August 2018 / Published online: 10 August 2018

(c) Fondazione Annali di Matematica Pura ed Applicata and Springer-Verlag GmbH Germany, part of Springer Nature 2018

\begin{abstract}
We introduce a CR-invariant class of Lorentzian metrics on a circle bundle over a threedimensional CR structure, which we call FRT metrics. These metrics generalise the Fefferman metric, allowing for more control of the Ricci curvature, but are more special than the shearfree Lorentzian metrics introduced by Robinson and Trautman.Our main result is a criterion for embeddability of three-dimensional CR structures in terms of the Ricci curvature of the FRT metrics in the spirit of the results by Lewandowski et al. (Class Quantum Gravity 7(11):L241L246, 1990) and also Hill et al. (Indiana Univ Math J 57(7):3131-3176, 2008. https://doi. org/10.1512/iumj.2008.57.3473).
\end{abstract}

Keywords Embeddability of CR manifolds · Shearfree null congruence $\cdot$ Lorentzian manifolds

Mathematics Subject Classification $32 \mathrm{~V} 30 \cdot 53 \mathrm{~B} 30 \cdot 83 \mathrm{C} 05$

\section{Introduction}

A three-dimensional strictly pseudoconvex almost CR structure is a three-dimensional manifold $M$ with a contact distribution $D$ and a smooth field of endomorphisms $J_{x}: D_{x} \rightarrow D_{x}$ such that $J_{x}^{2}=-$ id. Such structures naturally occur on strictly pseudoconvex real hypersurfaces $M$ in $\mathbb{C}^{2}$ where

$$
D_{x}=T_{x} M \cap J_{x} T_{x} M
$$

and $J_{x}$ is the canonical complex structure on $T_{x} \mathbb{C}^{2}$. The (local) embeddability (or realisability) problem asks whether there exists a (local) embedding $\iota: M \rightarrow \mathbb{C}^{2}$ of the abstract $\mathrm{CR}$ manifold $M$ such that the $\mathrm{CR}$ structures on $\iota M$ induced by $\iota$ and by $\mathbb{C}^{2}$ coincide. This problem

Gerd Schmalz

schmalz@une.edu.au

Masoud Ganji

mganjia2@une.edu.au

1 School of Science and Technology, University of New England, Armidale, NSW 2351, Australia 
is equivalent to finding two functionally independent CR functions, that is, solutions to the complex linear PDE

$$
\bar{\partial} f=0,
$$

where $\bar{\partial}=X+\mathrm{i} J X$ for some non-vanishing section $X$ of $D$.

While Eq. (1) has sufficiently many solutions for real-analytic CR structures (see e.g. [8]) to make them embeddable, it is well known that for almost all non-analytic CR structures only constant CR functions exist $[9,14,15]$.

It has been known to physicists since the 1960s (see e.g. [4]) that the existence of a non-constant CR function on $M$ is equivalent to the existence of a Lorentzian metric $g$ on $\mathcal{M}=M \times \mathbb{R}$ that satisfies two properties

(a) The fundamental vector field $k=\partial_{r}$ of the line bundle $M \times \mathbb{R}$ is null and shearfree with respect to $g$, that is, $g(k, k)=0$ and

$$
\mathcal{L}_{k} g=\rho g+\theta \vee \psi,
$$

where $\mathcal{L}$ is the Lie derivative, $\rho$ is a function on $\mathcal{M}, \theta=g(k, \cdot)$ and $\psi$ is some 1 -form on $\mathcal{M}$.

(b) The complexified Ricci tensor of $g$ vanishes on $\alpha$-planes, i.e. $g$ is partially Ricci flat. (See (10) for the precise definitions.)

It turns out that the existence of non-constant solutions to (1) is not sufficient to guarantee embeddability of the CR structure [10]. A remarkable theorem by Jacobowitz [7] provides a criterion for embeddability of CR structures in terms of the canonical bundle (see Definition 5.2). Below we reformulate Jacobowitz's more general result for three-dimensional CR structures.

Theorem 1.1 (Jacobowitz 1989) [7] Let $(M, D, J)$ be a CR structure. Suppose that near some point $p \in M$ the $C R$ structure has a CR function $\phi$ with $d \phi \wedge d \bar{\phi} \neq 0$. If the canonical bundle associated with the CR structure has a non-vanishing closed section, then the $C R$ structure is embeddable near $p$.

Notice that the converse statement of Theorem 1.1 is also true: for an embeddable CR structure, we have two functionally independent, hence non-constant, coordinate CR functions $\zeta, \eta$. The 2 -form $d \zeta \wedge d \eta$ gives a non-vanishing closed section of the canonical bundle.

Lewandowski et al. [12] and also Hill et al. [3] prove a series of embeddability results in terms of shearfree congruences of Lorentzian spaces. In particular, they show that a CR structure is embeddable if and only if it admits a lift to a partially Ricci flat Lorentzian space $(\mathcal{M}, g)$ with a shearfree congruence $k$ as above, and a Maxwell field aligned with $k$. This is closely related to Jacobowitz's Theorem 1.1, since the existence of such Maxwell field is equivalent to the existence of a non-vanishing closed section of the canonical bundle from Theorem (1.1) (see [16]).

In this paper, we introduce a family of Lorentzian metrics on a circle bundle over the CR manifold $M$. This family is more general than the conformal class of Fefferman metrics but more special than the family of Lorentzian metrics that admit a shearfree congruence, introduced by Robinson and Trautman [17,18]. We call these metrics FRT metrics (for Fefferman, Robinson, Trautman). Our main result is the following embeddability criterion.

Theorem 1.2 A strictly pseudoconvex $C R$ structure $(M, D, J)$ is locally embeddable if and only if it admits an FRT metric for which the complexified Ricci curvature vanishes on the $\alpha$-planes. 
Notice that the special choice of the metrics allows us to drop the assumption of the existence of a non-vanishing closed section of the canonical bundle. In order to give a selfcontained proof, we recapitulate techniques used in [3] and references therein.

\section{CR structures and the Fefferman metric}

Let $(M, D, J)$ be a three-dimensional CR structure. We assume that $(M, D, J)$ is strictly pseudoconvex, i.e. for any (local) non-vanishing section $X$ of $D,[X, J X] \notin D$. For any choice of $X$, we have an adapted complex frame $\partial, \bar{\partial}, \partial_{0}$, where

$$
\partial=X-\mathrm{i} J X, \quad \partial_{0}=\mathrm{i}[\partial, \bar{\partial}]=-2[X, J X] .
$$

The complex vector field $\partial=X-\mathrm{i} J X$ spans the + i-eigendistribution $D^{1,0}$ of $J$ in $D \otimes \mathbb{C}$. We denote the dual coframe to $\left(\partial, \bar{\partial}, \partial_{0}\right)$ by $(\mu, \bar{\mu}, \lambda)$. Strict pseudoconvexity of $M$ translates to

$$
d \lambda \wedge \lambda \neq 0
$$

Our choice implies

$$
\begin{aligned}
d \lambda & =\mathrm{i} \mu \wedge \bar{\mu}+c \mu \wedge \lambda+\bar{c} \bar{\mu} \wedge \lambda, \\
d \mu & =\alpha \mu \wedge \lambda+\beta \bar{\mu} \wedge \lambda,
\end{aligned}
$$

where $c, \alpha, \beta$ are complex-valued functions on $M$. Any other distinguished frame $\left(\partial^{\prime}, \bar{\partial}^{\prime}, \partial_{0}^{\prime}\right)$ and coframe $\left(\mu^{\prime}, \bar{\mu}^{\prime}, \lambda^{\prime}\right)$ express through the original frame and coframe by

$$
\begin{aligned}
\partial^{\prime} & =\mathrm{e}^{-\tau-\mathrm{i} \theta} \partial, & & \partial_{0}^{\prime}=\mathrm{e}^{-2 \tau}\left(\partial_{0}-h \partial-\bar{h} \bar{\partial}\right) \\
\mu^{\prime} & =\mathrm{e}^{\tau+\mathrm{i} \theta}(\mu+h \lambda), & & \lambda^{\prime}=\mathrm{e}^{2 \tau} \lambda
\end{aligned}
$$

where $\tau$ and $\theta$ are real-valued functions and

$$
\begin{aligned}
& h=-\mathrm{i} \bar{\partial}(\tau+\mathrm{i} \theta), \quad \alpha^{\prime}=\mathrm{e}^{2 \tau}\left(\alpha-\partial_{0}(\tau+\mathrm{i} \theta)+h \partial(\tau+\mathrm{i} \theta)+\partial h+h c\right), \\
& c^{\prime}=\mathrm{e}^{-\tau-\mathrm{i} \theta}(c-2 \mathrm{i} \bar{h}+\partial(\tau+\mathrm{i} \theta)) .
\end{aligned}
$$

Recall that the Fefferman metric is a conformal class of Lorentzian metrics defined on the circle bundle $\mathcal{M}=D^{1,0} / \mathbb{R}^{+}$. Using a distinguished coframe $(\mu, \lambda)$ and the trivialisation $\left.\mathcal{M} \ni m\right|_{p}=\left.\mathrm{e}^{-t-\mathrm{i} \rho} \partial\right|_{p} \mapsto(p, \rho)$ where $\rho \in[0,2 \pi)$, a representative of the Fefferman metric is defined by the simple formula

$$
g_{F}=\mu \bar{\mu}+\lambda\left(\frac{2}{3} d \rho-\frac{\mathrm{i}}{3} c \mu+\frac{\mathrm{i}}{3} \bar{c} \bar{\mu}-\left(\frac{\partial \bar{c}+\bar{\partial} c}{12}-\frac{\mathrm{i}(\alpha-\bar{\alpha})}{4}\right) \lambda\right)
$$

where we kept the notations $\mu, \bar{\mu}, \lambda$ for their pullbacks under the circle bundle projection, and $\alpha$ and $c$ are as above (see [11]). The CR invariance of the Fefferman metric means that a change in the distinguished coframe $(\mu, \bar{\mu}, \lambda)$ causes only a conformal change in $g_{F}$ by the factor $\mathrm{e}^{2 \tau}$.

Denote by $\tilde{\mathcal{M}}$ the natural lift of $\mathcal{M}$ to a line bundle. It will be convenient in the computations below to rescale the coordinate $\rho$ on $\tilde{\mathcal{M}}$ to $r=\frac{2 \rho}{3}$. Then, the change in the coframe $(\lambda, \mu)$ induces the change

$$
r^{\prime}=r-\frac{2}{3} \theta
$$


in the trivialisation of $\tilde{\mathcal{M}}$. Denote the quotient bundle of the (rescaled) line bundle $\tilde{\mathcal{M}}$ mod $2 \pi$ by $\mathcal{M}^{\frac{3}{2}}$. Since the Fefferman metric is invariant with respect to the principle $\mathbb{R}$-bundle action, it projects to any $S^{1}$-bundle with arbitrary period. In particular, it is well defined on $\mathcal{M}^{\frac{3}{2}}$.

It is known that the Weyl tensor of the Fefferman metric has rank (at most) one [11]. However, in general, the Ricci curvature cannot be controlled and, except for very special cases (see [2,5]), one cannot find (partially) Ricci flat or Einstein representatives of the conformal Fefferman metric.

\section{FRT metrics}

We generalise the Fefferman metric by introducing functional parameters $x$ and $H$ in addition to the conformal factor. This allows us to impose conditions on the Ricci curvature that cannot be satisfied by the Fefferman metric. We will see later that it is more natural to define the FRT metrics on the circle bundle $\mathcal{M}^{\frac{3}{2}}$ rather than the Fefferman bundle $\mathcal{M}$.

Definition 3.1 Let $(M, D, J)$ be a CR structure and $\mathcal{M}^{\frac{3}{2}}$ as above. For any choice of a distinguished coframe $(\mu, \lambda)$ and the induced trivialisation of $\mathcal{M}^{\frac{3}{2}}$, we define the family of FRT metrics on $\mathcal{M}^{\frac{3}{2}}$ by

$$
g=2 P^{2}[\mu \bar{\mu}+\lambda(d r+W \mu+\bar{W} \bar{\mu}+H \lambda)],
$$

where $W=\mathrm{i} x \mathrm{e}^{-\mathrm{i} r}-\frac{\mathrm{i}}{3} c$. Here, $P \neq 0, H$ are real-valued functions on $\mathcal{M}^{\frac{3}{2}}$ and $x$ is a complex-valued function on $M$.

It is an important feature of the family of shearfree metrics (8) and of the conformal family of Fefferman metrics that they are CR invariant, i.e. they do not depend on the choice of the pair $(\mu, \lambda)$. We show that this is also true for the family of FRT metrics.

\section{Theorem 3.2 The family of FRT metrics is CR invariant.}

Proof Under the frame change (4) and the induced change in the trivialisation (7), the FRT metric changes as follows:

$$
\begin{aligned}
g^{\prime}= & 2 P^{\prime 2}\left[\mu^{\prime} \overline{\mu^{\prime}}+\lambda^{\prime}\left(d r^{\prime}+\left(\mathrm{i} x^{\prime} \mathrm{e}^{-\mathrm{i} r^{\prime}}-\frac{\mathrm{i}}{3} c^{\prime}\right) \mu^{\prime}+\left(-\mathrm{i} \overline{x^{\prime}} \mathrm{e}^{\mathrm{i} r^{\prime}}+\frac{\mathrm{i}}{3} \bar{c}^{\prime}\right) \overline{\mu^{\prime}}+H^{\prime} \lambda^{\prime}\right)\right] . \\
g^{\prime}= & 2|f|^{2} P^{\prime 2}\left[\mu \bar{\mu}+\bar{h} \mu \lambda+h \bar{\mu} \lambda+|h|^{2} \lambda^{2}+\lambda\left(d r-\frac{2}{3} d \theta+f\left(\mathrm{i} x^{\prime} \mathrm{e}^{\mathrm{i} \frac{2}{3} \theta} \mathrm{e}^{-\mathrm{i} r}-\frac{\mathrm{i}}{3 f}(c-2 \mathrm{i} \bar{h}+\partial \log f)\right) \mu\right.\right. \\
& +\bar{f}\left(-\mathrm{i} \overline{x^{\prime}} \mathrm{e}^{-\mathrm{i} \frac{2}{3} \theta} \mathrm{e}^{\mathrm{i} r}+\frac{\mathrm{i}}{3 \bar{f}}(\bar{c}+2 \mathrm{i} h+\bar{\partial} \log \bar{f})\right) \bar{\mu}+\left(f h\left(\mathrm{i} x^{\prime} \mathrm{e}^{\mathrm{i} \frac{2}{3} \theta} \mathrm{e}^{-\mathrm{i} r}-\frac{\mathrm{i}}{3} c^{\prime}\right)\right. \\
& \left.\left.\left.+\bar{f} \bar{h}\left(-\mathrm{i} \overline{x^{\prime}} \mathrm{e}^{-\mathrm{i} \frac{2}{3} \theta} \mathrm{e}^{\mathrm{i} r}+\frac{\mathrm{i}}{3} \bar{c}^{\prime}\right)+|f|^{2} H^{\prime}\right) \lambda\right)\right] . \\
= & 2 P^{2}\left[\mu \bar{\mu}+\lambda\left(d r+\left(\mathrm{i} x \mathrm{e}^{-\mathrm{i} r}-\frac{\mathrm{i}}{3} c\right) \mu+\left(\frac{1}{3} \bar{h}-\frac{\mathrm{i}}{3} \partial \log f-\frac{2}{3} \partial \theta\right) \mu+\left(-\mathrm{i} \bar{x} \mathrm{e}^{\mathrm{i} r}+\frac{\mathrm{i}}{3} \bar{c}\right) \bar{\mu}\right.\right. \\
& \left.\left.+\left(\frac{1}{3} h+\bar{\partial} \log \bar{f}-\frac{2}{3} \bar{\partial} \theta\right) \bar{\mu}+H \lambda\right)\right] . \\
= & 2 P^{2}\left[\mu \bar{\mu}+\lambda\left(d r+\left(\mathrm{i} x \mathrm{e}^{-\mathrm{i} r}-\frac{\mathrm{i}}{3} c\right) \mu+\left(-\mathrm{i} \bar{x} \mathrm{e}^{\mathrm{i} r}+\frac{\mathrm{i}}{3} \bar{c}\right) \bar{\mu}+H \lambda\right)\right],
\end{aligned}
$$


where

$$
\begin{aligned}
& f=\mathrm{e}^{\tau+\mathrm{i} \theta}, \quad P=\mathrm{e}^{\tau} P^{\prime}, \quad x=\mathrm{e}^{\tau+\mathrm{i} \frac{5}{3} \theta} x^{\prime}, \\
& H=\mathrm{e}^{2 \tau} H^{\prime}+|h|^{2}+\mathrm{e}^{\tau+\mathrm{i} \theta} h\left(\mathrm{i} x^{\prime} \mathrm{e}^{-\mathrm{i} r^{\prime}}-\frac{\mathrm{i}}{3} c^{\prime}\right)+\mathrm{e}^{\tau-\mathrm{i} \theta} \bar{h}\left(-\mathrm{i} \overline{x^{\prime}} \mathrm{e}^{\mathrm{i} r^{\prime}}+\frac{\mathrm{i}}{3} \overline{c^{\prime}}\right)-\frac{2}{3} \partial_{0} \theta .
\end{aligned}
$$

\section{Lorentzian geometry and $\alpha$-planes}

Let $(\mathcal{M}, g)$ be a four-dimensional Lorentzian manifold equipped with a foliation into integral curves of a non-vanishing null vector field $k$. We have the following canonical objects

(i) the 1 -form $\theta=g(k, \cdot)$,

(ii) the distribution $k^{\perp}=\{X \in \Gamma(T \mathcal{M}): g(X, k)=0\}$,

(iii) the distribution of screen spaces $S:=k^{\perp} / k$.

Proposition 4.1 On each screen space $S_{x}$, there are two canonical complex structures $J_{x}$ and $-J_{x}$.

Proof The restriction of $g$ to $k^{\perp}$ is a degenerate metric with kernel $k$ and induces a Euclidean metric on $S_{x}$. Since $S_{x}$ is two dimensional, the Euclidean metric induces complex structures of rotation by $\frac{\pi}{2}$ in either orientation.

Choose one of the two complex structures on $S$. Then, $\mathbb{C} \otimes S$ splits into its eigenspaces $S^{1,0} \oplus S^{0,1}$. Let

$$
\pi: \mathbb{C} \otimes k^{\perp} \rightarrow \mathbb{C} \otimes S
$$

be the canonical projection map. The subspaces $K^{1,0}, K^{0,1}$ of $\mathbb{C} \otimes k^{\perp}$ defined by

$$
K^{1,0}=\pi^{-1} S^{1,0}, \quad K^{0,1}=\pi^{-1} S^{0,1}
$$

are called $\alpha$-planes and $\beta$-planes, respectively. Notice that changing the orientation used in the definition of $J$ results in interchanging the $\alpha$-planes and the $\beta$-planes.

Clearly,

$$
K^{1,0} \cap K^{0,1}=k, \quad K^{1,0}+K^{0,1}=\mathbb{C} \otimes k^{\perp} .
$$

Definition 4.2 We say that the complexified Ricci tensor of $g$ vanishes on the $\alpha$-planes $K^{1,0}$ if $\left.R i c\right|_{K^{1,0}}=0$, i.e.

$$
\operatorname{Ric}\left(X_{1}, X_{2}\right)=0 \quad \forall X_{1}, X_{2} \in K^{1,0} .
$$

Notice that vanishing of the complexified Ricci tensor on $\alpha$-planes is equivalent to its vanishing on $\beta$-planes. Hence, the definition above does not depend on the choice of $J$.

Definition 4.3 Let $\mathcal{M}$ be a four-dimensional manifold equipped with a Lorentzian metric $g$ and a non-vanishing null vector field $k$. A complex frame $\left(e_{1}, e_{2}, \ell, k\right)$ is called adapted to $(g, k)$ if $e_{1}$ is a section of $\alpha$-planes, $e_{2}=\bar{e}_{1}$ (and, hence, is a section of $\beta$-planes), and

$$
g\left(e_{1}, e_{2}\right)=1, \quad g(\ell, \ell)=0, \quad g(\ell, k)=1, \quad g\left(\ell, e_{1}\right)=\overline{g\left(\ell, e_{2}\right)}=0 .
$$

Proposition 4.4 A four-dimensional Lorentzian manifold $(\mathcal{M}, g)$ with a non-vanishing null vector field $k$ possesses (locally) a complex adapted frame. 
Proof Let $\ell$ be a null vector field such that $g(\ell, k)=1$. Choose a unit vector field $\varepsilon_{1} \in k^{\perp} \cap \ell^{\perp}$. Choose $\varepsilon_{2} \in k^{\perp}$ such that $\pi \varepsilon_{2}=J \pi\left(\varepsilon_{1}\right)$ and $g\left(\varepsilon_{2}, \ell\right)=0$. Now, set

$$
e_{1}=\frac{1}{\sqrt{2}}\left(\varepsilon_{1}-\mathrm{i} \varepsilon_{2}\right), \quad e_{2}=\frac{1}{\sqrt{2}}\left(\varepsilon_{1}+\mathrm{i} \varepsilon_{2}\right) \text {. }
$$

It follows that the $\alpha$-planes are spanned by $\left(e_{1}, k\right)$ and the $\beta$-planes are spanned by $\left(e_{2}, k\right)$. Now, vanishing of the Ricci curvature on $\alpha$-planes is equivalent to

(i) $R_{11}=\operatorname{Ric}\left(e_{1}, e_{1}\right)=\overline{\operatorname{Ric}\left(e_{2}, e_{2}\right)}=0$,

(ii) $R_{14}=\operatorname{Ric}\left(e_{1}, k\right)=\overline{\operatorname{Ric}\left(e_{2}, k\right)}=0$,

(iii) $R_{44}=\operatorname{Ric}(k, k)=0$.

For the dual complex coframe $\left(\theta^{1}, \theta^{2}, \theta^{3}, \theta^{4}\right)$ to an adapted complex frame, $\bar{\theta}^{1}=\theta^{2}$ vanishes on $\alpha$-planes, $\theta^{3}=g(k, \cdot)$ and $g=2\left(\theta^{1} \theta^{2}+\theta^{3} \theta^{4}\right)$. The Gram matrix for both $g$ and its dual $g^{-1}$ with respect to an adapted frame and coframe is:

$$
\left(\begin{array}{llll}
0 & 1 & 0 & 0 \\
1 & 0 & 0 & 0 \\
0 & 0 & 0 & 1 \\
0 & 0 & 1 & 0
\end{array}\right) .
$$

A direct computation shows that the shearfreeness condition (2) is equivalent to

$$
d \theta^{3} \wedge \theta^{1} \wedge \theta^{3}=0, \quad d \theta^{1} \wedge \theta^{1} \wedge \theta^{3}=0 .
$$

See, e.g. [3] for details.

Below we cite a version of the celebrated Goldberg-Sachs theorem [1,3,6], which is a useful tool for computing certain components of the Weyl tensor of the Lorentzian metric $g$ :

$$
C_{i j k l}=R_{i j k l}+\frac{1}{6} R\left(g_{i k} g_{l j}-g_{i l} g_{k j}\right)+\frac{1}{2}\left(g_{i l} R_{k j}-g_{i k} R_{l j}+g_{j k} R_{l i}-g_{j l} R_{k i}\right),
$$

where $R_{i j k l}$ is the Riemann curvature, $R_{k j}$ is the Ricci curvature and $R$ is the scalar curvature. The following quantities are called Weyl scalars:

$$
\Psi_{0}=C\left(k, e_{1}, k, e_{1}\right)=C_{4141}, \quad \Psi_{1}=C\left(k, l, k, e_{1}\right)=C_{4341} .
$$

Theorem 4.5 (Goldberg-Sachs theorem [1,3]) Suppose that a four-dimensional manifold $\mathcal{M}$ is equipped with a Lorentzian metric $g$ and a shearfree null vector field $k$, as above. Also assume that the complexified Ricci curvature of $g$ vanishes on $\alpha$-planes, i.e. $R_{11}=$ $R_{14}=R_{44}=0$ with respect to an adapted coframe $\left(\theta^{1}, \theta^{2}, \theta^{3}, \theta^{4}\right)$. Then, the Weyl scalars $\Psi_{0}=\Psi_{1}=0$.

The next lemma plays a crucial role in finding a CR function. It reduces the existence of a non-vanishing CR function to the existence of a certain complex-valued 1-form. Such 1-form can be obtained from the Levi-Civita connection form of the associated Lorentzian metric, if the Ricci curvature vanishes on $\alpha$-planes. The proof is based on Frobenius's theorem. For a detailed proof, see e.g. [3].

Lemma 4.6 [3] Let $\varphi$ be a smooth complex-valued 1-form defined locally in $\mathbb{R}^{n}, n \geq 3$, such that $\varphi \wedge \bar{\varphi} \neq 0$. Then

$$
d \varphi \wedge \varphi=0
$$

if and only if there exist smooth complex functions $\zeta$ and $h$ such that

$$
\varphi=h d \zeta, \quad d \zeta \wedge d \bar{\zeta} \neq 0 .
$$




\section{FRT metrics and the embedding of three-dimensional CR structures}

Before we prove the main theorem of this paper, we collect some more ingredients. First we compute the Levi-Civita connection 1-forms, $\Gamma_{j}^{i}$ with respect to an adapted frame. Notice that due to (3) we have

$$
\left[\partial, \partial_{0}\right]=-\alpha \partial-\bar{\beta} \bar{\partial}-c \partial_{0}, \quad\left[\bar{\partial}, \partial_{0}\right]=-\beta \partial-\bar{\alpha} \bar{\partial}-\bar{c} \partial_{0}
$$

Now in terms of the coframe $\left(\theta^{1}, \theta^{2}, \theta^{3}, \theta^{4}\right)$ with

$$
\theta^{1}=P \mu, \quad \theta^{2}=P \bar{\mu}, \quad \theta^{3}=P \lambda, \quad \theta^{4}=P(d r+W \mu+\bar{W} \bar{\mu}+H \lambda),
$$

the metric (8) becomes

$$
g=2 \theta^{1} \theta^{2}+2 \theta^{3} \theta^{4} .
$$

The dual frame $\left(e_{1}, e_{2}, e_{3}, e_{4}\right)$ to $\left(\theta^{1}, \theta^{2}, \theta^{3}, \theta^{4}\right)$ takes the form

$$
e_{1}=\frac{1}{P}\left(\partial-W \partial_{r}\right), \quad e_{2}=\frac{1}{P}\left(\bar{\partial}-\bar{W} \partial_{r}\right), \quad e_{3}=\frac{1}{P}\left(\partial_{0}-H \partial_{r}\right), \quad e_{4}=\frac{1}{P} \partial_{r} .
$$

The commutators of the frame fields (15) evaluate to

$$
\begin{aligned}
{\left[e_{1}, e_{2}\right]=} & \left(\frac{\bar{\partial} P}{P^{2}}-\bar{W} \frac{P_{r}}{P^{2}}\right) e_{1}+\left(-\frac{\partial P}{P^{2}}+W \frac{P_{r}}{P^{2}}\right) e_{2}-\frac{\mathrm{i}}{P} e_{3}+\left(-\frac{\mathrm{i} H}{P}+W_{2}-\bar{W}_{1}\right) e_{4} \\
{\left[e_{1}, e_{3}\right]=} & \left(\frac{\partial_{0} P}{P^{2}}-H \frac{P_{r}}{P^{2}}-\frac{\alpha}{P}\right) e_{1}-\frac{\bar{\beta}}{P} e_{2}+\left(-\frac{\partial P}{P^{2}}+W \frac{P_{r}}{P^{2}}-\frac{c}{P}\right) e_{3} \\
& +\left(-\frac{c H}{P}+W_{3}-H_{1}-\frac{\alpha W}{P}-\frac{\bar{\beta} \bar{W}}{P}\right) e_{4} \\
{\left[e_{1}, e_{4}\right]=} & \frac{P_{r}}{P^{2}} e_{1}+\left(-\frac{\partial P}{P^{2}}+W \frac{P_{r}}{P^{2}}+\frac{W_{r}}{P}\right) e_{4} \\
{\left[e_{3}, e_{4}\right]=} & \frac{P_{r}}{P^{2}} e_{3}+\left(-\frac{\partial_{0} P}{P^{2}}+H \frac{P_{r}}{P^{2}}+\frac{H_{r}}{P}\right) e_{4},
\end{aligned}
$$

where the subscripts $1,2,3$ in the above expressions denote derivation with respect to the corresponding frame field (15). For example, $H_{1}$ means $\frac{1}{P}\left(\partial H-W H_{r}\right)$.

Now by using these commutator relations and Cartan's structure equations

$$
d \theta^{i}+\Gamma_{k}^{i} \wedge \theta^{k}=0
$$


for the metric (14), we find the connection forms listed as follows:

$$
\begin{aligned}
& \Gamma_{4}^{1}=\left(\frac{\mathrm{i}}{2 P}+c_{14}^{1}\right) \theta^{1}+\frac{1}{2}\left(c_{23}^{3}+c_{24}^{4}\right) \theta^{3}, \\
& \Gamma_{1}^{1}=-c_{12}^{2} \theta^{1}-c_{12}^{1} \theta^{2}+\frac{1}{2}\left(c_{23}^{2}-c_{13}^{1}-c_{12}^{4}\right) \theta^{3}+\frac{\mathrm{i}}{2 P} \theta^{4} \\
& \Gamma_{4}^{4}=c_{34}^{4} \theta^{3}+c_{34}^{3} \theta^{4}-\frac{1}{2} c_{23}^{3} \theta^{2}-\frac{1}{2} c_{13}^{3} \theta^{1}+\frac{1}{2} c_{14}^{4} \theta^{1}+\frac{1}{2} c_{24}^{4} \theta^{2} \\
& \Gamma_{1}^{3}=\left(\frac{\mathrm{i}}{2 P}-c_{24}^{2}\right) \theta^{2}-\frac{1}{2}\left(c_{13}^{3}+c_{14}^{4}\right) \theta^{3} \\
& \Gamma_{1}^{4}=-c_{13}^{2} \theta^{1}-\frac{1}{2}\left(c_{12}^{4}+c_{23}^{2}+c_{13}^{1}\right) \theta^{2}-c_{13}^{4} \theta^{3}-\frac{1}{2}\left(c_{14}^{4}+c_{13}^{3}\right) \theta^{4} \\
& \Gamma_{3}^{1}=\frac{1}{2}\left(-c_{12}^{4}+c_{13}^{1}+c_{23}^{2}\right) \theta^{1}+c_{23}^{1} \theta^{2}+c_{23}^{4} \theta^{3}+\frac{1}{2}\left(c_{24}^{4}+c_{23}^{3}\right) \theta^{4},
\end{aligned}
$$

where $c_{m n}^{k}$ are the structure constants defined by $\left[e_{m}, e_{n}\right]=c_{m n}^{k} e_{k}$. We also notice that $d g_{i j}=\Gamma_{i j}+\Gamma_{j i}$ and hence

$$
\Gamma_{2}^{1}=\Gamma_{11}=0 \quad \text { and } \quad \Gamma_{4}^{3}=\Gamma_{33}=0 .
$$

Remark 1 Note that because of the choice of the coframe (13), complex conjugation of the connection forms interchanges the indices 1 and 2 and keeps the indices 3, 4 unchanged, for example, $\bar{\Gamma}_{4}^{1}=\Gamma_{4}^{2}$.

In the proposition below, we compute the Ricci components of the FRT metric.

Proposition 5.1 Let $g$ be an FRT metric (8) on $\mathcal{M}^{\frac{3}{2}}$ associated with a CR manifold $M$ that admits a non-constant CR function $\zeta$. Let $(\mu=d \zeta, \lambda)$ be a coframe for $M$ and $R_{i k}$ the components of the Ricci curvature with respect to an adapted frame. Then,

(i) $R_{44}=0$ is equivalent to

$$
P=\frac{a}{\cos \left(\frac{r+s}{2}\right)}
$$

where $a, s$ are arbitrary $r$-independent real functions.

(ii) $R_{24}=0$ is equivalent to

$$
\partial \log a^{2}+\mathrm{i} \partial s-2 x \mathrm{e}^{\mathrm{i} s}=-\frac{2 c}{3},
$$

(iii) $R_{22}=0$ if and only if the equation

$$
\partial t+t(c-t)=0
$$

is satisfied where

$$
t=c+\partial \log a^{2}-x \mathrm{e}^{\mathrm{i} s} .
$$

For an alternate coframe $\left(\mu^{\prime}, \lambda^{\prime}\right)$, the function $t$ changes to

$$
t^{\prime}=\mathrm{e}^{-\tau-\mathrm{i} \theta}(t-\mathrm{i} \bar{h}) .
$$


Proof To verify that the condition $R_{44}=0$ is equivalent to the function $P$ having the form (19), we first notice that $R_{44}=2 R_{414}^{1}$. We now consider the Cartan's structure equation for the 1 -form $\Gamma_{4}^{1}$

$$
d \Gamma_{4}^{1}+\Gamma_{k}^{1} \wedge \Gamma_{4}^{k}=R_{4 k \ell}^{1} \theta^{k} \wedge \theta^{\ell}, \quad k<\ell,
$$

which simplifies to

$$
d \Gamma_{4}^{1}+\Gamma_{1}^{1} \wedge \Gamma_{4}^{1}+\Gamma_{4}^{1} \wedge \Gamma_{4}^{4}=R_{4 k \ell}^{1} \theta^{k} \wedge \theta^{\ell},
$$

since $\Gamma_{2}^{1}=\Gamma_{4}^{3}=0$. Substituting the 1 -forms $\Gamma_{4}^{1}, \Gamma_{1}^{1}, \Gamma_{4}^{4}$ given by (16), (17), (18) into (24) and inspecting the coefficient of the 2 -form $\theta^{1} \wedge \theta^{4}$ in (24), we get the differential equation

$$
-4 P P_{r r}+8 P_{r}^{2}+P^{2}=0 .
$$

The general solution of the differential equation (25) has the form (19).

A similar argument shows that $R_{24}=0$ is equivalent to (20).

To show that $R_{22}=0$ is equivalent to (21), we first notice that, due to $d \mu=0$, the structure functions $\alpha, \beta$ vanish. Then, by similar arguments as above, $R_{22}=0$ becomes equivalent to the differential equation (21).

To verify (23), we first notice that

$$
P^{\prime}=\mathrm{e}^{-\tau} P=\frac{a \mathrm{e}^{-\tau}}{\cos \left(\frac{r+s}{2}\right)}=\frac{a^{\prime}}{\cos \left(\frac{r^{\prime}+s^{\prime}}{2}\right)}
$$

for all $r$ and $r^{\prime}=r-\frac{2}{3} \theta$. It follows $a^{\prime}=\mathrm{e}^{-\tau} a$ and $s^{\prime}=\frac{2}{3} \theta+s$. Therefore,

$$
\begin{aligned}
t^{\prime}= & c^{\prime}+\partial^{\prime} \log a^{\prime 2}-x^{\prime} \mathrm{e}^{\mathrm{i} s^{\prime}}=\mathrm{e}^{-\tau-\mathrm{i} \theta}(c-2 \mathrm{i} \bar{h}+\partial(\tau+\mathrm{i} \theta))+\mathrm{e}^{-\tau-\mathrm{i} \theta}\left(\partial \log a^{2}-2 \partial \tau\right) \\
& -\mathrm{e}^{-\tau-\frac{5 \mathrm{i}}{3} \theta} x \mathrm{e}^{\mathrm{i} s+\frac{2 \mathrm{i}}{3} \theta} \\
= & \mathrm{e}^{-\tau-\mathrm{i} \theta}\left(c-2 \mathrm{i} \bar{h}+\partial(\tau+\mathrm{i} \theta)+\partial \log a^{2}-\partial(\tau+\mathrm{i} \theta)+\mathrm{i} \bar{h}-x \mathrm{e}^{\mathrm{i} s}\right)=\mathrm{e}^{-\tau-\mathrm{i} \theta}(t-\mathrm{i} \bar{h}) .
\end{aligned}
$$

Another important ingredient is Jacobowitz's theorem (1.1), which uses the notion of the canonical bundle.

Definition 5.2 Let $(M, D, J)$ be a CR structure and $D^{\mathbb{C}}=D^{1,0} \oplus D^{0,1}$ the eigenspace decomposition of $J$. The canonical bundle $\mathcal{K}$ over $M$ is the complex line bundle of complexvalued 2-forms with kernel $D^{0,1}$, i.e.

$$
\mathcal{K}=\left\{\phi \in \Lambda^{2}(M) \otimes \mathbb{C} \mid \phi(X, \cdot)=0 \quad \forall X \in D^{0,1}\right\} .
$$

If the CR structure $(M, D, J)$ is given by the coframe $(\lambda, \mu)$, then the canonical bundle is spanned by $\lambda \wedge \mu$. Using this representation, the existence of a closed non-vanishing section can be reformulated as a $\bar{\partial}$-problem.

Proposition 5.3 A CR structure $(M,[(\lambda, \mu)])$ admits locally a non-vanishing closed section of the canonical bundle if and only if the $\bar{\partial}$-problem

$$
\bar{\partial} \log \psi=-\bar{c},
$$

has a solution. Here, $c$ is the structure function from (3). 
Proof Taking into account (3),

$$
d(\psi \mu \wedge \lambda)=\bar{\partial} \psi \bar{\mu} \wedge \mu \wedge \lambda-\bar{c} \psi \mu \wedge \bar{\mu} \wedge \lambda=(\bar{\partial} \psi+\bar{c} \psi) \bar{\mu} \wedge \mu \wedge \lambda
$$

vanishes if and only if (26) is satisfied with non-vanishing $\psi$.

While we do not assume the existence of a non-vanishing closed section of the canonical bundle a priori, we show that this is a consequence of our assumptions.

We are now ready to prove our main theorem.

Theorem 5.4 A three-dimensional $C R$ structure $(M, D, J)$ is (locally) embeddable if and only if there exists an associated circle bundle $\mathcal{M}^{\frac{3}{2}}$ with an FRT metric $g$ whose complexified Ricci tensor vanishes on the distribution of $\alpha$-planes.

Proof Let $M$ be a CR structure with coframe $(\mu, \lambda)$ and let $g$ be an FRT metric defined by (8) on $\mathcal{M}^{\frac{3}{2}}$ for which $R_{22}=R_{24}=R_{44}=0$. We consider the connection 1-form

$$
\Gamma_{24}=\Gamma_{4}^{1}=\sigma \theta^{1}+\rho \theta^{3}
$$

from (16) where

$$
\sigma=\frac{\mathrm{i}}{2 P}+\frac{P_{r}}{P^{2}}, \quad \rho=-\frac{\bar{\partial} P}{P^{2}}+\frac{\bar{W} P_{r}}{P^{2}}-\frac{\bar{c}}{2 P}+\frac{\bar{W}_{r}}{P} .
$$

Clearly, $\sigma \neq 0$ and therefore, the form $\Gamma_{24} \neq 0$. Moreover,

$$
\Gamma_{24} \wedge \bar{\Gamma}_{24} \neq 0,
$$

since

$$
\Gamma_{24} \wedge \bar{\Gamma}_{24}=|\sigma|^{2} \theta^{1} \wedge \theta^{2} \bmod \theta^{3} .
$$

On the other hand, the conditions of the Goldberg-Sachs Theorem (4.5) with respect to the shearfree vector field $\partial_{r}$ are satisfied and therefore

$$
\Psi_{0}=C_{4141}=R_{1414}=0, \quad \Psi_{1}=C_{4341}=\frac{1}{2}\left(R_{4341}+R_{1421}\right)=0 .
$$

It follows

$$
C_{4242}=\overline{C_{4141}}=0, \quad C_{4342}=\overline{C_{4341}}=0
$$

and furthermore, using the symmetries of the Riemann curvature

$$
R_{i j k \ell}=R_{k \ell i j}, \quad R_{i j k \ell}=-R_{j i k \ell}=-R_{i j \ell k},
$$

we get

$$
R_{2424}=0, \quad R_{2434}+R_{2412}=0 .
$$

Since

$$
R_{44}=2 R_{2414}, \quad R_{22}=2 R_{2423}, \quad R_{24}=R_{2412}-R_{2434},
$$

where $R_{i j}=R_{i k j}^{k}$ and $R_{i j k \ell}=g_{i m} R_{j k \ell}^{m}$, this shows that the conditions $R_{44}=R_{22}=R_{24}=0$ are equivalent to

$$
R_{2414}=R_{2423}=R_{2412}-R_{2434}=0 .
$$


Combining (28) and (29) yields

$$
R_{2412}=R_{2424}=R_{2414}=R_{2423}=R_{2434}=0 .
$$

Therefore, Cartan's structure equation (24) for the connection 1-form $\Gamma_{24}=\Gamma_{4}^{1}$ becomes

$$
d \Gamma_{24}-\left(\Gamma_{12}+\Gamma_{34}\right) \wedge \Gamma_{24}=R_{24 k \ell} \theta^{k} \wedge \theta^{\ell}=R_{2413} \theta^{1} \wedge \theta^{3} .
$$

Wedging the equation above with $\Gamma_{24}$ and taking into account that $\Gamma_{24}$ is a linear combination of $\theta^{1}$ and $\theta^{3}$, given by (27), we conclude that

$$
d \Gamma_{24} \wedge \Gamma_{24}=0 .
$$

Now we can apply Lemma (4.6) for the 1 -form $\Gamma_{24}$ and deduce that locally there exist complex functions $h \neq 0, \zeta$ such that

$$
\Gamma_{24}=h d \zeta \text { with } d \zeta \wedge d \bar{\zeta} \neq 0 .
$$

Wedging the equation

$$
h d \zeta=\Gamma_{24}=P(\sigma \mu+\rho \lambda)
$$

by $\lambda \wedge \mu$ shows that

$$
d \zeta \wedge \lambda \wedge \mu=0
$$

Restricting the function $\zeta$ to the CR manifold $M$, considered as a section $\{r=0\}$ of $\mathcal{M}^{\frac{3}{2}}$, gives a $\mathrm{CR}$ function there.

Now we may assume that $\mu=d \zeta$. Since vanishing of the Ricci tensor on the $\alpha$-planes does not depend on the choice of an adapted frame, the conditions $R_{44}=R_{24}=R_{22}=0$ are still satisfied.

We consider the two cases $t \equiv 0$ and $t \not \equiv 0$, where $t$ is defined by (22). In the first case, it follows from equation (20) that

$$
\frac{4}{3} c=-\partial \log a^{2}+\mathrm{i} \partial s
$$

and hence

$$
\partial \log \left(a^{\frac{3}{2}} \mathrm{e}^{-\frac{3}{4} \mathrm{i} s}\right)=-c .
$$

Therefore, Eq. (26) has a solution $\psi=a^{\frac{3}{2}} \mathrm{e}^{\frac{3}{4} \mathrm{i} s}$ and, by Proposition 5.3, the canonical bundle has a nowhere vanishing closed section. Now, by Theorem 1.1, the CR structure is embeddable and, in particular, there exists a second CR function that is functionally independent from $\zeta$.

In the second case, if $t$ is not identically 0 , we replace the complex coframe 1 -form $\mu$ by another exact form $\mu^{\prime}$ as follows. Consider

$$
\varphi=\mu+\mathrm{i} \bar{t} \lambda
$$

Since $R_{22}=0$ we have

$$
d \varphi \wedge \varphi=\mathrm{i}(\bar{\partial} \bar{t}+\bar{t}(\bar{c}-\bar{t})) \mu \wedge \bar{\mu} \wedge \lambda=0 .
$$

Also $\varphi \wedge \bar{\varphi} \neq 0$ holds because

$$
\varphi \wedge \bar{\varphi}=\mu \wedge \bar{\mu}-\mathrm{i} t \mu \wedge \lambda-\mathrm{i} \bar{t} \bar{\mu} \wedge \lambda .
$$


Thus, the 1-form $\varphi$ satisfies the conditions of the Lemma (4.6). Consequently, there exist complex-valued functions $b \neq 0, \eta$ such that

$$
\varphi=\mu+\mathrm{i} \bar{t} \lambda=b d \eta .
$$

Clearly,

$$
d \eta \wedge d \bar{\eta}=\frac{1}{|b|^{2}} \varphi \wedge \bar{\varphi} \neq 0
$$

It follows from the definition of $\eta$ and $\varphi$ that $d \eta$ is a linear combination of $\mu$ and $\lambda$ and hence

$$
d \eta \wedge \lambda \wedge \mu=0,
$$

that is, $\eta$ is a CR function. Now we switch to the coframe $\left(\mu^{\prime}=d \eta, \lambda^{\prime}\right)$ for which, because of (23), $t^{\prime} \equiv 0$ everywhere. This reduces the second case to the first case and proves embeddability of $M$.

For the proof of the converse statement, we assume that the CR structure $M$ with adapted coframe $(\mu=d \zeta, \lambda)$ is embeddable. Then, the canonical bundle contains a nonzero closed section, i.e. there exists a nonzero complex function $\psi$ such that

$$
\partial \log \bar{\psi}=-c .
$$

We define real functions $a, s$ and a complex function $x$ as follows:

$$
\log a^{2}=\frac{4}{3} \operatorname{Re}(\log \bar{\psi}), \quad s=-\frac{4}{3} \operatorname{Im}(\log \bar{\psi}), \quad x=\mathrm{e}^{-\mathrm{i} s}\left(c+\partial \log a^{2}\right) .
$$

The metric defined by

$$
g=2 P^{2}[\mu \bar{\mu}+\lambda(d r+W \mu+\bar{W} \bar{\mu}+H \lambda)],
$$

where

$$
P=\frac{a}{\cos \left(\frac{r+s}{2}\right)}, \quad W=\mathrm{i} x \mathrm{e}^{-\mathrm{i} r}-\frac{\mathrm{i}}{3} c,
$$

and $H$ is any real function defined on $\mathcal{M}^{\frac{3}{2}}$, is an FRT metric for $(M, \mu, \lambda)$ and, due to Proposition (5.1), $R_{44}=R_{24}=R_{22}=0$ is satisfied.

Acknowledgements The authors wish to express their gratitude to Howard Jacobowitz and Jerzy Lewandowski for inspiring and useful discussions.

\section{References}

1. Gover, A.R., Hill, C.D., Nurowski, P.: Sharp version of the Goldberg-Sachs theorem. Ann. Mat. Pura Appl. (4) 190(2), 295-340 (2011)

2. Čap, A., Gover, A.R.: CR-tractors and the Fefferman space. Indiana Univ. Math. J. 57(5), 2519-2570 (2008)

3. Hill, C.D., Lewandowski, J., Nurowski, P.: Einstein's equations and the embedding of 3-dimensional CR manifolds. Indiana Univ. Math. J. 57(7), 3131-3176 (2008). https://doi.org/10.1512/iumj.2008.57.3473

4. Debney, G.C., Kerr, R.P., Schild, A.: Solutions of the Einstein and Einstein-Maxwell equations. J. Math. Phys. 10, 1842-1854 (1969)

5. Leitner, F.: On transversally symmetric pseudo-Einstein and Fefferman-Einstein spaces. Math. Z. 256(2), 443-459 (2007)

6. Goldberg, J.N., Sachs, R.K.: A theorem on Petrov types. Acta Phys. Pol. 22, 13-23 (1962) 
7. Jacobowitz, H.: The canonical bundle and realizable CR hypersurfaces. Pac. J. Math. 127(1), 91-101 (1987)

8. Jacobowitz, H.: An Introduction to CR Structures. Mathematical Surveys and Monographs, vol. 32. American Mathematical Society, Providence (1990)

9. Jacobowitz, H., Trèves, F.: Nonrealizable CR structures. Invent. Math. 66(2), 231-249 (1982)

10. Rosay, J.-P.: New examples of non-locally embeddable CR structures (with no nonconstant CR distributions). Ann. Inst. Fourier (Grenoble) 39(3), 811-823 (1989). (English, with French summary)

11. Lewandowski, J.: On the Fefferman class of metrics associated with a three-dimensional CR space. Lett. Math. Phys. 15(2), 129-135 (1988)

12. Lewandowski, J., Nurowski, P., Tafel, J.: Einstein's equations and realizability of CR manifolds. Class. Quantum Gravity 7(11), L241-L246 (1990)

13. Lee, J.M.: Introduction to Smooth Manifolds. Graduate Texts in Mathematics, vol. 218, 2nd edn. Springer, New York (2013)

14. Nirenberg, L.: Lectures on linear partial differential equations, American Mathematical Society, Providence, R.I., 1973. Expository Lectures from the CBMS Regional Conference held at the Texas Technological University, Lubbock, Tex., 22-26 May 1972; Conference Board of the Mathematical Sciences Regional Conference Series in Mathematics, No. 17

15. Nirenberg, L.: On a Problem of Hans Lewy, Fourier Integral Operators and Partial Differential Equations (Colloque International, Universite de Nice, 1974). Lecture Notes in Mathematics, vol. 459, pp. 224-234. Springer, Berlin (1975)

16. Robinson, I.: Null electromagnetic fields. J. Math. Phys. 2, 290-291 (1961). https://doi.org/10.1063/1. 1703712

17. Robinson, I., Trautman, A.: New theories in physics. In: Pokorski, S., Ajduk, Z., Trautman, A. (eds.) Optical Geometry, pp. 454-497. World Scientific, Singapore (1989)

18. Trautman, A.: Robinson manifolds and Cauchy-Riemann spaces. Class. Quantum Gravity 19(2), R1-R10 (2002). https://doi.org/10.1088/0264-9381/19/2/201 